\title{
Detection of circulating tumor cells after surgery for stage III and IV squamous cell carcinoma of the head and neck
} \author{
and Dolivet $\mathrm{G}^{1,2}$ \\ ${ }^{1}$ Universite de Lorraine, CNRS, CRAN, F-54000 Nancy, France \\ ${ }^{2}$ Institut de Cancerologie de Lorraine, 6 avenue de Bourgogne, 54511 Vandoeuvre-lès-Nancy, France \\ ${ }^{3}$ CHRU Nancy 54511 Vandoeuvre les Nancy France, France \\ ${ }^{4}$ TECHNOPORT 9, avenue des hauts-Fourneaux L-4362 Esch/ Alzette Belval Luxembourg, France
}

Mastronicola $\mathrm{R}^{1,2 *}$, Cortese $\mathrm{S}^{2}$, Beulque $\mathrm{E}^{2}$, Xanglie $\mathrm{WU}^{3}$, Roch $\mathrm{M}^{3}$, Perna $\mathrm{M}^{4}$, Salleron $\mathrm{J}^{2}$, Merlin $\mathrm{JL}^{1,2}$, Faure $\mathrm{G}^{3}$, Munier $\mathrm{J}^{2}$, Bolotine $\mathrm{L}^{1,2}$

\begin{abstract}
In this study, we focused mainly on the metastatic process related to surgery in squamous cell carcinoma of the upper aerodigestive tract. We attempted to detect isolated cells (CTCs) of head and neck squamous cell carcinoma (SCCHN) in blood stream before, during and after surgery. With this aim, we realized a prospective study analyzing the impact of surgery on the CTC level during a period of 9 days. Two widely used techniques were used in this study : PCR in real time and Cell Search.
\end{abstract}

\begin{abstract}
Abbreviations: SCCHN: Squamous cell carcinoma head and neck; CTC: Circulating tumor cells; EGFR: Epidermal growth factor receptor; EpCAM: Epithelial cell adhesion molecule; EMT: Epithelialmesenchymal transition; CEA: Carcinoembryonic antigen; CK 18,19: Cytokeratin 18,19; Ef3: E74-like 3; PVA: Pemfigus vulgaris antigen, SCCA: Squamous cell carcinoma antigen; EphB4: Tyrosine kinase erythropoietin producing hepatocellular; RT-PCR: Reverse transcription-polymerase chain reaction; CTM: Circulating tumor Microemboli.
\end{abstract}

\section{Background}

The impact of surgery on cancer biology is a large issue from cell dissemination to immunosuppression. Metastatic disease remains one of the leading causes of death in SCCHN cancers. However, the incidence of distant metastasis in SCCHN remains low when compared to other types of cancer such as breast or lung cancer. Currently, there is an urgent need for new and better prognostic markers to improve the prediction of locoregional recurrence and distant metastasis in SCCHN patients.

Metastasis can occur during surgery as a cascade of events that starts with tumor cells detaching from their primary tumor site after partial or complete Epithelial-Mesenchymal phenotype transition. The Epithelial-Mesenchymal transition (EMT) is a complex morphogenetic process where epithelial cells lose their characteristics and gain mesenchymal properties during embryogenesis or progression of cancer. This step is followed by cells breaching into the blood or the lymphatic system. Some cells may undergo apoptosis throughout circulation, due to venous and arterial stress shear, immunesurveillance and high velocities. The surviving cells can circulate as single cells called Circulating Tumor Cells (CTCs) or as cell clusters, termed Circulating tumor Microemboli (CTM), which have a higher metastatic potential. CTCs can remain dormant in distant tissues, thus forming micro metastases that may progress into secondary, overt macro metastases [1].

The EMT process is induced and regulated by effectors such as growth factors (TGF $\beta$ ) but also by hypoxia. It is characterized by the loss of epithelial markers (E-cadherin, cytokeratins, occludin, and ZO-1), gain of mesenchymal markers (N-cadherin, vimentin, and fibronectin) and high expression of MMPs, which help cancer cells grow and spread.

Previous studies have shown that visually confirmed presence of CTCs in peripheral blood of patients with SCCHN was predictive of cancer recurrence and/or cancer related mortality [2]. As a matter of fact, the detection of CTCs has predictive value in patients with SCCHN, mainly on the tumor progression. The absence of CTCs may be considered as an indicator for disease-free survival. However, CTC status in patients with SCCHN appears to be not associated with TNM disease stage and nodal involvement [3].

The ability to detect Circulating Tumor Cells may have an impact on the prognosis and treatment of cancer patients. Currently, this topic is poorly addressed. The most studied approach to detect CTCs include polymerase chain reaction (PCR), microfluidic chips, immunomagnetic enrichment, flow cytometry and laser scanning cytometry. The sensitivity and specificity of these methods for CTC detection depend on the markers used. An important issue is the markers choice since SCCHN has many mutual markers with other cell types [4-6].

In this study, we focused mainly on the metastatic process related to surgery in squamous cell carcinoma of the upper aerodigestive

${ }^{*}$ Correspondence to: Romina Mastronicola, PhD, MD Institut de cancerologie de Lorraine, 6 avenue de Bourgogne 54511 Vandœuvre les Nancy France, France, E-mail: r.mastronicola@nancy.unicancer.fr

Received: March 01, 2019; Accepted: April 19, 2019; Published: May 03, 2019 
tract. We attempted to detect isolated cells (CTCs) of head and neck squamous cell carcinoma (SCCHN) in blood stream before, during and after surgery. With this aim, we realized a prospective study analyzing the impact of surgery on the CTC level during a period of 9 days. Two widely used techniques were used in this study : PCR in real time and Cell Search.

\section{Methods}

\section{Patient's characteristics}

Forty patients were enrolled in this study. The study was approved by Ethics Committee of patient's protection ( ${ }^{\circ} \mathrm{CPP}: 2010-\mathrm{A} 00586-33$ ). All enrolled patients signed the consent. They all underwent head and neck surgery for SCCHN at the Institut de Cancerologie de Lorraine (Nancy, France) between April 2014 and April 2015. The patients underwent radiological diagnosis, were assigned as having operable SCCHN and hadn't prior cancer treatments (surgery, radiotherapy or chemotherapy). The median patient's age was 66 (37 to 84). Concerning patient's cancer stage: 2 of them were stage II, 23 were stage III and 15 were stage IV.

Venous blood samples were collected at three time points (preoperative day-1, intraoperative and postoperative day-7). We detected CTCs using two different methods: quantitative PCR in real time and CellSearch system.

\section{Disseminated tumor cells detection: Cell search system}

The CellSearch system was used to detect Disseminated Tumor Cells. In the absence of detection kit for head and neck cancer, we used CellSearch epithelial Kit and EGFR. CellSearch Circulating Tumor Cell Kit is intended for the enumeration of circulating tumor cells of epithelial origin in whole blood [7-9].

The CellSearch system includes 2 components: The Auto-Prep System and the CellTracks Analyzer (Veridex LLC, USA). The AutoPrep System selected CTCs and detected them using automated and standardized immunomagnetic cell enrichment. The Auto-Prep System separated the cells using a 2-stage magnetic separation procedure: the first step consists of enrichment and is followed by detection step. In this way, immunomagnetic selection was performed using ferrofluid particles coated with EpCAM antibodies. This step yielded unbound cells and plasma exclusion, and tumor cells enrichment. Then, permeabilization buffer (used for cytokeratins staining) and fluorescent reagent were added to the remaining EpCAM-positive cells. Fluorescent reagents included DAPI for nuclear labeling, anti-cytokeratin (CK 8, $18,19)$ to detect epithelial cells and anti-CD45 antibodies conjugated with allophycocyanin for leukocyte exclusion.

The CellTracks Analyzer is a semi-automated fluorescence microscope which is connected to analysis software. This software detects, captures and displays images of four colors fluorescent cells on the computer screen. The operator confirms sCTCs presence and manually counts them. The Disseminated Tumor Cell responds to several criteria: positive nuclear staining (DAPI: purple), cytokeratin positive expression (green) and CD-45 negative staining.

Typical image of a Circulating Tumor Cell is displayed on the Figure 1. The nucleus is colored in red, while green color stands for the EGFR. This cell is negative for CD 45 and positive for CK markers, both confirming its tumor nature (8-18-19).

\section{Disseminated tumor cells detection: RT-PCR}

Reverse Transcription-Polymerase Chain Reaction (RT-PCR) is the most sensitive currently available technique for mRNA detection and quantitation.

In our study, we used iScript Advanced cDNA Synthesis Kit [9]. This kit is an enhanced formulation composed of nuclease-free water, reverse transcriptase enzyme and a reaction mix with dNTPs and oligo(dt). Once the reaction Mix is prepared, we used a thermal cycler (Eppendorf, Le Pecq, France) consisting in successive steps (Table 1).

The first step, called denaturation, allows the separation of the nucleic acids' double chain. The second one, called hybridization, allows the binding of the complimentary DNA bases. The last one, DNA elongation, facilitates the polymerization carried out by DNA polymerase.

The RT-PCR uses fluorescent reporter molecules to monitor the production of amplification products during each cycle of the PCR reaction. When the DNA is in the log linear phase of amplification, the amount of fluorescence increased above the background. In this study, we used SYBR Green (iQ, SYBR Green Supremix, BIO-RAD) as a fluorescent reporter. SYBR Green fluoresces only when bound it is to dsDNA. The increase in fluorescence corresponds to the increase in double stranded DNA.

In our study, we used 9 markers for quantitative RT-PCR: EphB4, Elf3, CEA, CK 18, CK19, Ep-CAM, EGFR, PVA, SCCA and GAPDH (reference gene).

\section{Statistical methods}

The digital parameters were described by mean value, median value, first quartile and third quartile, minimum and maximum values through Boxplot. Qualitative parameters are frequency and percentage. The markers normality of distribution was investigated with ShapiroWilks test. Some parameters had to be changed (logarithmic transformation) to restore the normality of distribution.

Markers' progression was studied at three times point (preoperative, intraoperative and postoperative) using a mixed linear model. This way, we took account of measurements repetition in any individual patient. Two post-hoc analyses were completed in order to compare times side-by-side. A Bonferroni correction allowed correcting alpha's

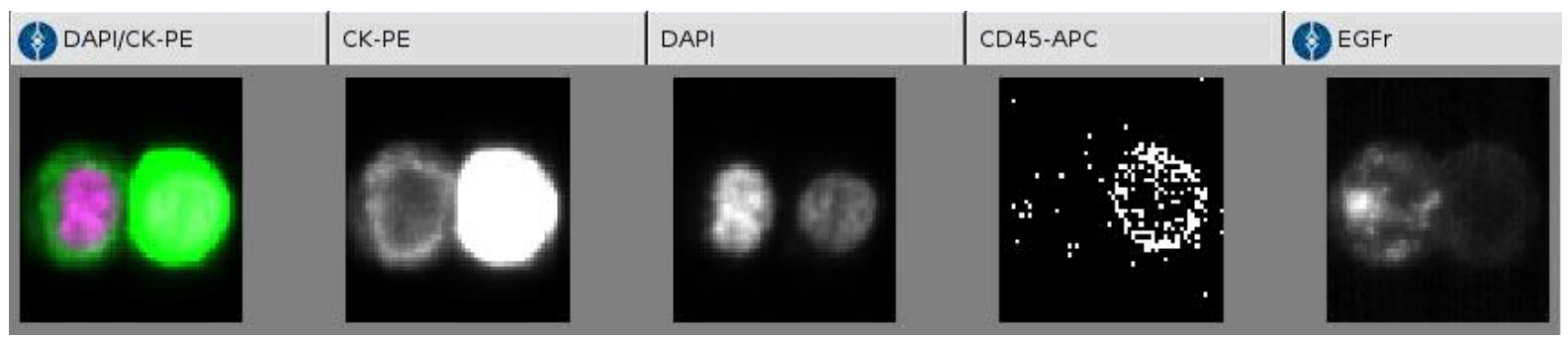

Figure 1. Images of CTC cell search 
Table 1. Steps RT-PCR iScript

\begin{tabular}{|c|c|c|}
\hline Step & Timing & Temperature \\
\hline 1 & $5 \mathrm{~min}$ & $25^{\circ} \mathrm{C}$ \\
\hline 2 & $30 \mathrm{~min}$ & $42^{\circ} \mathrm{C}$ \\
\hline 3 & $5 \mathrm{~min}$ & $85^{\circ} \mathrm{C}$ \\
\hline 4 & Until complete cooling & $4{ }^{\circ} \mathrm{C}$ \\
\hline
\end{tabular}

risk increment. This analysis was made either on gross values, either on transformed values (using logarithmic function), or stored values (Conover method).

An exploratory analysis was conducted to investigate the influence of clinical parameters (localization, vascular embolus, nodal involvement and transmission) on markers' values. For this use, each marker was compared at each time among clinical parameter. A MannWhitney test was performed concerning vascular embolus, nodal involvement and transmission whereas we used a Kruskal-Wallis test for localization.

A two-sided $\mathrm{p}<0.05$ was considered statistically significant. All analyses were performed with v9.3 (SAS, Cary, NC, USA).

\section{Results}

Table 2 demonstrates the results of biomarker detection obtained with two techniques. The RT-PCR shows significant results $(\mathrm{p}<0.05)$ for EphB4, CEA, CK 18 and Ep-CAM (Shapiro-Wilks test). Other tested markers were not significantly expressed.

Results obtained with CellSearch system using Epithelial Kit were non-significant $(p>0.05)$, while the expression of EGFR was statistically significant $(p=0.01)$. The kinetic of EGFR expression was further analyzed with Boxplot illustrating the incidence of surgery (Figure 2). In all studied patients we observed the significant decrease in EGFR ( $\mathrm{p}<$ 0.05 ) expression already two hours after surgery that remained stable within 7 days. The same profile was observed with Cell Search-assessed Epithelia Kit expression; however, the data were not significant $(\mathrm{p}>0.1)$ (Figure 3).

Among four RT-PCR-assessed biomarkers, which demonstrated a statistically significant expression (Table 2), we were particularly interested with EphB4. The pivotal role of EphB in H\&N cancers is acknowledged . Figure 4 (a) displays the individual patients' variations in EphB4 expression. We can note strong variations in EphB4 expression, probably coinciding with tumor cells under-population. Boxplot presentation (Figure 4b) demonstrated 1.5 times increase in the marker expression at $2 \mathrm{~h}$ after the surgery onset and return to the basal (pre-operative level) 7 days later. Other statistically significant markers analyzed with RT-PCR (Table 2) provided the same profiles, however we did not depict them for the clarity of presentation.

\section{Discussion}

This study related to tumor cell dissemination during surgery allows us to state some objective facts. For the first time we have demonstrated that the surgical procedure for SCCHN stages III and IV is at the origin of CTCs in the blood and lymphatic vessels. Earlier, the preliminary investigations with small number of samples suggested that intraoperative tumor manipulations at surgery for primary breast and colorectal cancers induce tumor cell dissemination (12-14). Another study showed that radical surgery for prostate adenocarcinoma leads to frequent prostate cell spread preoperatively and during manipulation with the tumor (15). This spillage of prostatic cells showed a strong biological predictive power.
Two different techniques were employed in our study for monitoring of patients' blood: Cell Search System and RT-PCR. Cell Search provides information for the prognosis and monitoring of patients with breast, prostate or colorectal cancer . At the moment, this is the only technique approved by the FDA for CTCs detection. As follows from our results (Table 2), despite the absence of specific Kit for H\&N cancers, the CellSearch System seems practicable using Epithelial Kit and EGFR. In our recent study we already demonstrated the possibility to detect DTC with Cell Search in drains after neck dissection in 14 patients undergoing surgery for stages III and IV $\mathrm{H} \& \mathrm{~N}$ cancers [9]. Tumor cells were detected in the drains of $69 \%$

Table 2. Results Rt-PCR and CellSearch

\begin{tabular}{|c|c|c|c|}
\hline \multicolumn{2}{|c|}{ RT-PCR } & \multicolumn{2}{c|}{ CellSearch } \\
\hline EphB4 & $\mathrm{P}: 0,0003$ & EGFR & $\mathrm{P}: 0,01$ \\
\hline CEA & $\mathrm{P}: 0,006$ & & \\
\hline CK18 & $\mathrm{P}: 0,0011$ & & \\
\hline EP-CAM & $\mathrm{P}: 0,0299$ & & \\
\hline \multicolumn{2}{|c|}{ Non-significant RT-PCR } & Non-significant CellSearch \\
\hline Elf3 & $\mathrm{P}: 0,0764$ & CTC epithelial Kit & $\mathrm{P}: 0,17$ \\
\hline CK19 & $\mathrm{P}: 0,2525$ & & \\
\hline EGFR & $\mathrm{P}: 0,2158$ & & \\
\hline SCCA & $\mathrm{P}: 0,6551$ & & \\
\hline PVA & $\mathrm{P}: 0,0542$ & & \\
\hline
\end{tabular}

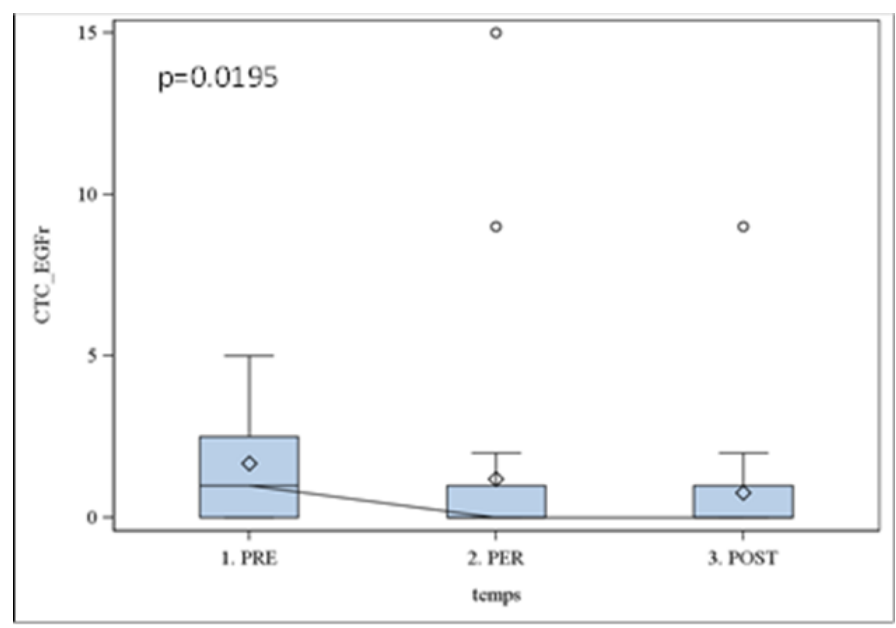

Figure 2. EGFR results using cell search

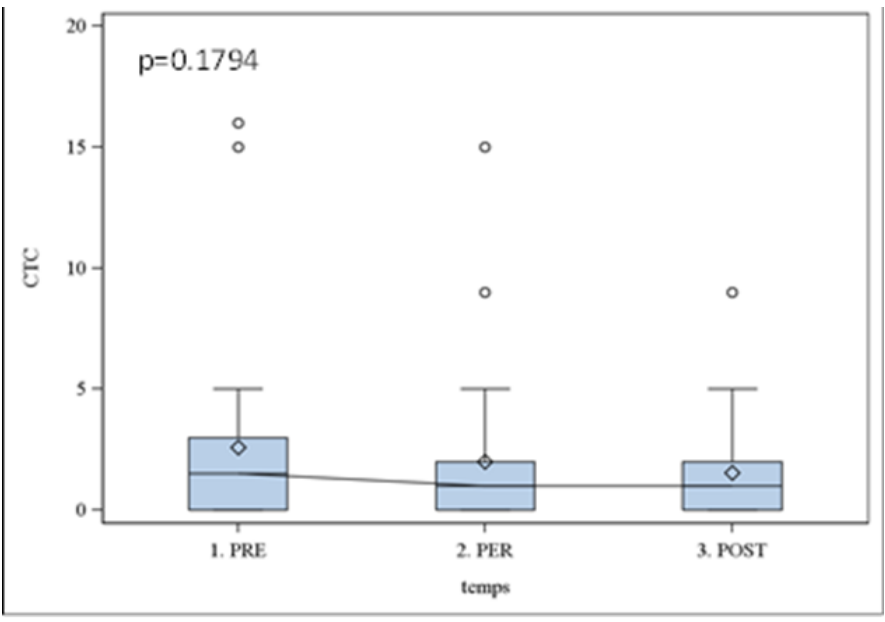

Figure 3. CTCs epithelial kit results with cell search 
of patients a few days after surgery. This study allowed us to suggest different therapeutic options in treatment of these patients. The main drawback of Cell Search system is that quantification of the results is hardly possible. On the contrary, RT-PCR provides quantitative aspect however a further optimization may be necessary for better detection sensitivity [8]. Recently, circulating microRNAs were considered as promising biomarkers for cancer diagnosis and prognosis [10]. MiRNAs may be considered as novel non-invasive biomarkers but the function of circulating miRNAs needs to be identified for their proper use in evidence-based medicine [11].

\section{Conclusion}

In our study four markers (EGFR, Ephb4, CEA, CK18) demonstrated significant variations related to the surgical procedure (Table 2). Those four markers were statistically significant increased compared to tumor cells which are already found in the area. It probably constitutes the tumor cell phenotype relevant to cells unleashed during surgery. Two hours after surgery, the expression of four markers, except of EGFR, is increased but seven to eight days after surgery the expression level becomes stable in blood flow (Figures 2-5). The increase in markers expression could be due either to proliferation of Circulating Tumor Cells or the empowerment of sleeper cells from their accumulation landmark.
There are specific marker evolution profiles for each patient and some markers show a particular profile (ex: EphB4 vs PVA) (Figures 4 and 5). Consequently, we demonstrated multitude of phenotypic patterns within individual patients. When the tested blood samples had a little or no sign of CTCs variation, we may consider that surgery hadn't impact on cells release (Table 2). If markers decreased, as in the case of EGFR, we may hypothesize that the tumor was completely eradicated.

Many questions are still pending on the study. It is not clear for the moment whether there is an interest for a medical treatment during the surgery in order to eliminate those disseminating cells. This question is largely conditioned by the relation between CTC and metastasis. As such, the potential clinical significance of tumor cells needs to be further investigated as their presence could affect presurgical and post-operative treatments. This issue is a subject of our ongoing investigations.

\section{Consent}

Our study is approval in ethics committed with $\mathrm{N}^{\circ} 2010-\mathrm{A} 00586-33 \mathrm{~N}^{\circ} \mathrm{CPP} 10.07 .03$ at Nancy le 10-03-2014.Written informed consent was obtained from the patients for publication of this study and any accompanying images. A copy of the written consent is available for review by request the Editor of this journal.

b

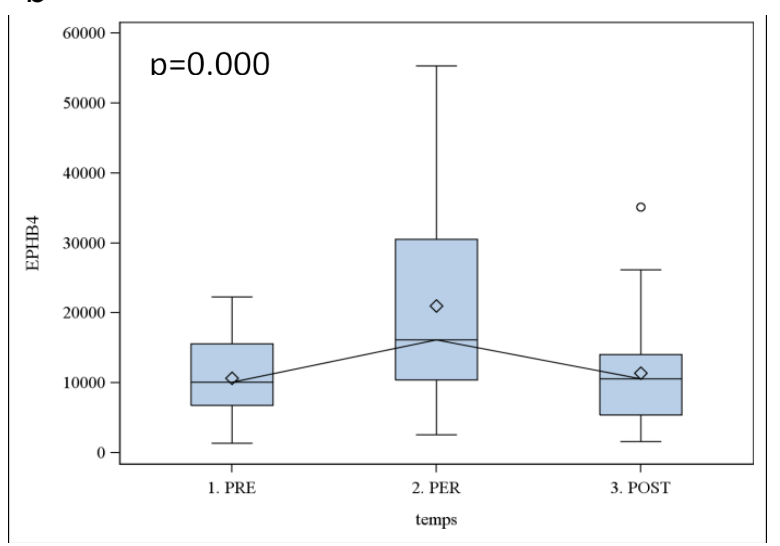

Figure 4. EphB4 (RT-PCR) expression in each patient (a) and boxplot (b) showing EphB4 variation

a

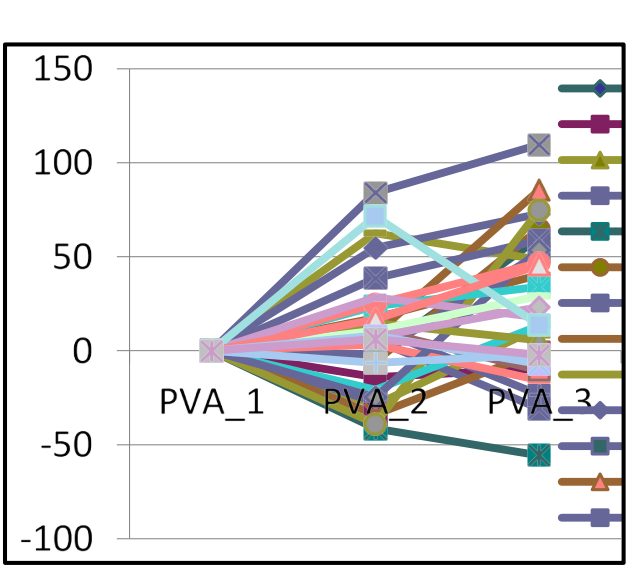

b

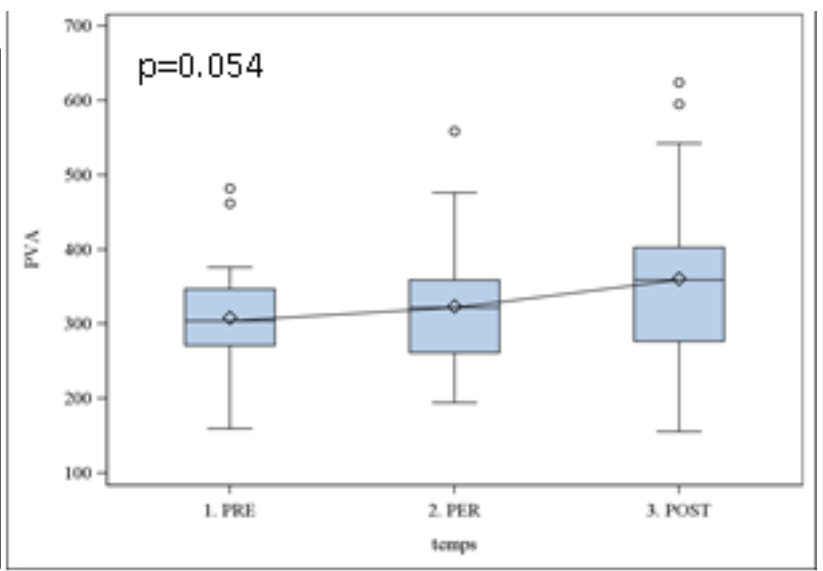

Figure 5. PVA (RT-PCR) expression in each patient (a) and boxplot (b) showing PVA variation 


\section{Funding}

Research Institut de cancerologie of Lorraine France.

\section{Authors' contributions}

$\mathrm{RM}, \mathrm{X} \mathrm{W}, \mathrm{JM}$ and JLM conducted the manipulations and data analysis; R M-SC-EB -MP-MR LB-JS was responsible for the clinical management; RM wrote the manuscript; R M,GF JLM and G D were in charge of the concept and design of the study; RM and G D reviewed and edited the manuscript extensively. All of the authors were involved in revision of the manuscript and approved the its final version of the manuscript.

\section{Competing interests}

The authors declare that they have no competing interests.

\section{References}

1. Kulasinghe C, Perry L, Jovanovic (2015) Circulating tumour cells in metastatic head and neck cancers. International Journal of Cancer 136: 2515-2523. [Crossref]

2. Wu X, Mastronicola R (2016) A rare case of extremely high counts of circulating tumor cells detected in a patient with an oral squamous cell carcinoma. BMC Cancer 16: 552. [Crossref]

3. Wang Z, Cui K, Xue Y (2014) Prognostic value of circulating tumor cells in patients with squamous cell carcinoma of the head and neck: a systemic review and metaanalysis. Springer Science 12: 4605-4613.

4. Jatana KR, Balasubramanian P, Lang JC, Yang L, Jatana CA, et al. (2010) Significance of circulating tumor cells in patients with squamous cell carcinoma of the head and neck: initial results. Arch Otolaryngol Head Neck Surg 136: 1274-1279. [Crossref]
5. M. Partridge, Brakenhoff R, Phillips E (2003) Detection of rare disseminated tumor cells identifies head and neck cancer patients at risk of treatment failure. Clinical cancer research 9: 5287-5294. [Crossref]

6. Balasubramanian P, Lang JC, Jatana KR (2012) Multiparameter Analysis, including EMT Markers, on Negatively Enriched Blood Samples from Patients with Squamous Cell Carcinoma of the Head and Neck. PLoS One 7: 11-33.

7. Nichols AC, Lowes LE, Szeto CC, Basmaji J, Dhaliwal S, et al. (2012) Detection of circulating tumor cells in advanced head and neck cancer using the CellSearch system. Head Neck 34: 1440-1444. [Crossref]

8. Shores CG, YIN X, Funkhouser W (2004) Clinical Evaluation of a new molecular method for detection of micrometastases in head and neck squamous cell carcinoma. Arch Otolaryngol Head Neck Surg 130. 937-942.

9. Mastronicola R, Berteau C, Tu Q (2016) Detection of disseminated tumor cells in aspirative drains after neck dissection. Eur Arch Otorhinolaryngol 273: 465-469. [Crossref]

10. Hui BY, Lenarduzzi M, Krushel T (2010) Comprehensive MicroRNA profiling for head and neck suqamous cell carcinoma. Clinical Cancer Research 16: 53-65

11. Kosaka N, Iguchi H, Ochiya T (2010) Circulating microRNA in body fluid: a new potential biomarker for cancer diagnosis and prognosis. Cancer Science 01: 23-28.

12. Wietz J, Kienle P, Lacroix J (1998) Dissemination of tumor cells in patients undergoing surgery for colorectal cancer. Clin Cancer Res 14: 21-32.

13. Weitz J, Herfarth C (2001) Surgical strategies and minimal residual disease detection. Semin Surg Oncol

14. Wiggers T, Jeekel J, Arends JW (1988) No-touch isolation technique in colon cancer: a controlled prospective trial. Br J Surg 75: 409-415.

15. Eschwege P, Moutereau S, Droupy S (2009) Prognostic value of prostate circulating cells detection in prostate cancer patients: a prospective study. BR J Cancer 100: 608610. [Crossref]

Copyright: (O2019 Mastronicola R. This is an open-access article distributed under the terms of the Creative Commons Attribution License, which permits unrestricted use, distribution, and reproduction in any medium, provided the original author and source are credited. 